\title{
Analysis for the extraction of phonological information using the vocal tract area
}

\author{
Akihide Fukatsu*, Izumi Hanazaki \\ Department of Robotics and Mechatronic, School of Science and technology for Future \\ Life, Tokyo Denki University, 5, Asahi-cho Senju Adachi-ku Tokyo, 120-8551,Japan \\ TEL:+81-3-5284-5609 ,FAX,:+81-3-5284-5698 \\ E-mail:fukatsu@hanalab.fr.dendai.ac.jp
}

\begin{abstract}
We can utter sounds by changing the shape of our vocal tract. The vocal tract characteristic has been analyzed by AR model for speech signals and the vocal tract area function can be estimated by PARCOR coefficients computed from the AR model. Phonological and personal information are included in the vocal tract area function. From this fact, the vocal tract area function can be considered to be available to voice synthesis or speaker recognition. In this paper, we will analyze the vocal tract area with the aim of recognition of phoneme of Japanese vowels.
\end{abstract}

\section{Introduction}

Speech signal is generated as a result of the vocal tract resonance vibration of the vocal cords as a sound source. Therefore, it is characterized by the shape of the vocal tract. Features of the vocal tract can be estimated by time series analysis of the speech signal by using the AR model. That is, the vocal tract area function representing the shape of the vocal tract can be derived from the coefficients of the AR model for speech signal. In recent years, research on the change of sound quality and speech synthesis, vocal tract area function have been used [1], [2], [3]. Vocal tract area function is considered to contain the phonological information and speaker information. They may be effective in speech recognition and speaker recognition. Therefore, in this paper, we will analyze the vocal tract area function derived from the AR model.

\section{Speech Signal Analysis based on AR Model}

\subsection{AR Model}

AR (autoregressive) model is represented by the following equation.

$$
x(n)+\sum_{k=1}^{p} a_{k} x(n-k)=e(n)
$$

where $x(n)\{n=0,1,2, \ldots\}$ is stationary signal, $p$ is AR model order, $a_{k}\{k=1,2, \ldots P\}$ is AR model coefficients and $e(n)$ is prediction error. In speech signal processing, AR model coefficients are estimated so as to fit $x(n)$ to the speech signal, and Burg's method is often adopted to estimate them.

\subsection{Burg's method}

In Burg's method, AR coefficients are estimated so as to minimize both of forward prediction error and backward prediction error represented in (2) and (3) respectively.

$$
\begin{gathered}
f e^{p}(n)=x(n)+\sum_{k=1}^{p} a_{k}{ }^{(p)} x(n-k) \\
(n=p, p+1, \ldots, N-1) \\
b e^{p}(n)=x(n-p)+\sum_{k=1}^{p} a_{k}{ }^{(p)} x(n-p+k) \\
(n=p, p+1, \ldots, N-1)
\end{gathered}
$$

In this way, estimates of the AR coefficients are sequentially updated through the reflection coefficient with respect to order. The reflection coefficient is shown in the following equation.

$$
\begin{gathered}
k_{p}=\frac{\sum_{n=p}^{N-1}\left[f e^{p-1}(n)\left(b e^{p-1}(n)\right]\right.}{\sum_{n=p}^{N-1}\left[f e^{p-1}(n)^{2}\right]+\sum_{n=p}^{N-1}\left[b e^{p-1}(n-1)^{2}\right]} \\
=-\frac{2 \sum_{n=p}^{N-1} f e^{p-1}(n) b e^{p-1}(n-1)}{\sum_{n=p}^{N-1}\left[\left\{f e^{p-1}(n)\right\}^{2}+\left\{b e^{p-1}(n-1)\right\}^{2}\right]}
\end{gathered}
$$


In Lattice structure representation of Burg's algorithm, the reflection coefficient $k_{p}$ is equivalent to the PARCOR coefficient.

\subsection{Vocal Tract Area}

Vocal tract can be approximated as series connection of acoustic tube with equal length and different radius. It is called Kelly's speech production model. Sound resonance phenomenon in the vocal tract is expressed by this model. Reflection of sound propagation in a vocal tract on the connecting face of sound tubes is equal to PARCOR coefficient in Kelly's model [4]. Vocal tract area function is represented by the following equation from PARCOR coefficient.

$$
A_{n}=\frac{1-k_{n}}{1+k_{n}} A_{n-1}(n=1,2, \ldots, p) \quad A_{0}=1
$$

where $A_{n}(n=1,2, \ldots, p)$ is vocal tract area function coefficients. Starting point of (5) equation, $A_{0}$, denotes lips. Vocal tract area function can be obtained by changing the ratio of the cross-sectional area adjacent to each other according to the reflection coefficient.

\subsection{Determination of $A R$ model order}

The order of AR model in coefficient estimation is usually selected on the AIC (Akaike's Information criteria) and FPE (Final prediction error). According to the comparison of Kelly's speech production model and AR model for speech signal, the number of acoustic tube corresponds to AR model order. Here, we are considering determination of AR model order suitable for estimating the vocal tract area function.

\subsubsection{Power spectrum}

Power spectrum of the speech signal is estimated as the frequency characteristics of the estimated AR model. The transfer function of AR model is shown in equation (6).

$$
H(z)=\frac{1}{1+\sum_{k=1}^{p} a_{k} z^{-k}}
$$

Then, power spectrum of the output of AR model $X_{p}(f)$ is written by next equation,

$$
X_{p}(f)=\frac{\sigma_{p}{ }^{2} \Delta t}{\left|1+\sum_{k=1}^{p} a_{k} e^{-(J 2 \pi f \Delta t k)}\right|^{2}}
$$

where $\Delta t$ is sampling frequency, $\sigma_{p}^{2}$ is prediction error variance, and $f$ denotes frequency. For example, the power spectrum of speech signal of Japanese vowel 'a' is shown figure 1.

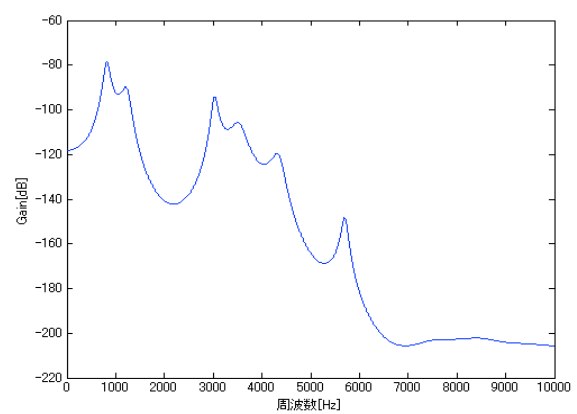

Fig.1 Power spectrum of speech signal, Japanese vowel 'a'

In this figure, we can see some peaks of power spectrum of speech signal. Their frequencies correspond to the resonance frequencies of the vocal tract and are own characteristic parameters of the signal, which are called formants. The resonance frequencies in low frequency band should be estimated accurately, when recognizing the vowels.

\subsubsection{Consideration in estimating power spectrum of the speech signal, on the relationship between the sampling frequency and AR model order}

We will investigate the relationship between sampling frequency and AR model when the suitable estimate of power spectrum of speech signal will be obtained, through the following experiments. Changing the order of the AR model and the sampling frequency as shown in Table 1, we will estimate the power spectrum of the audio signal of Japanese vowels, and evaluate the estimated value of the peak at the low frequency band. Then, we will choose the order AR model when the estimation value is obtained appropriately in each sampling frequency.

Table 1 Set of sampling frequency and AR model order

\begin{tabular}{l|l} 
Sampling frequency & $8,9,10,20,30,40,50[\mathrm{kHz}]$
\end{tabular} Speech signal "a", "i", "u", "e", "o"

AR model order from 1 to 170 Subject $\quad$ Male of twenties

Figure 2 shows analysis results for Japanese vowel "a". It shows the relationship between the sampling frequency and the lower and upper limits of the order of AR model where appropriate estimate is obtained. It also shows the power spectra estimated by using various AR model orders. 


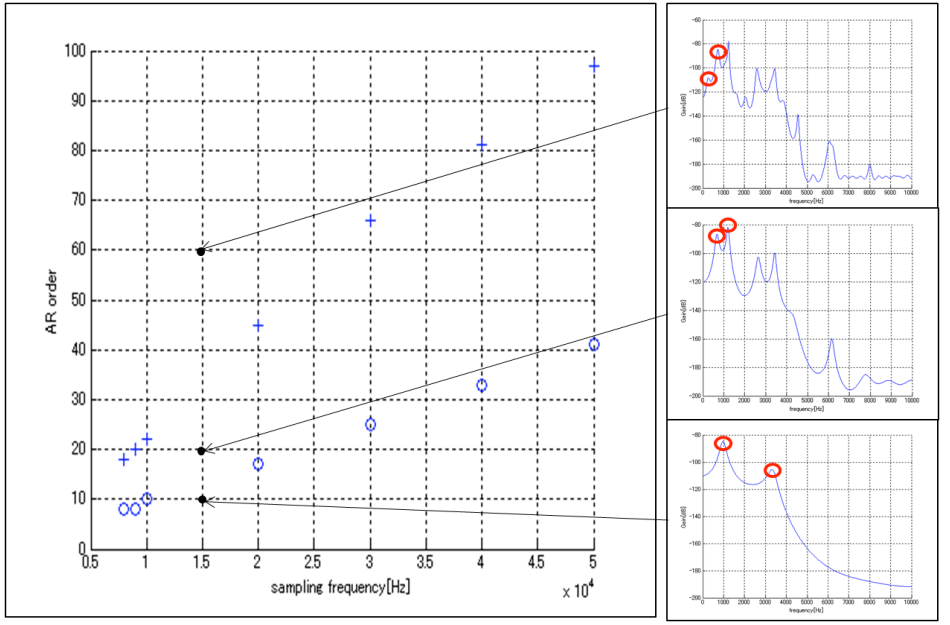

Fig 2 Relationship between proper AR model order and sampling frequency when speech signal, Japanese vowel "a", is analyzed by AR model

In case the order of AR model is set low, the formant cannot appear. In case the order is higher, the line sprits appear near the real formants. Namely even though recorded speech have the same sampling frequency setting, formant position is different depending on AR model order. Then, we consider selection of the suitable AR model order that corresponds to the sampling frequency. Table 2 shows the frequency band in which the formants of Japanese vowel signal should exist. Phoneme of Japanese vowel can be specified by the first and the second formant.

Table 2 Frequency band in which the first and the second formant should exist

\begin{tabular}{c|c}
\hline \hline Band of the first formant of "a" & $600 \sim 900[\mathrm{~Hz}]$ \\
Band of the second formant of "a" & $1000 \sim 1800[\mathrm{~Hz}]$ \\
Band of the first formant of "i" & $200 \sim 400[\mathrm{~Hz}]$ \\
Band of the second formant of "i" & $2000 \sim 2800[\mathrm{~Hz}]$ \\
Band of the first formant of "u" & $200 \sim 450[\mathrm{~Hz}]$ \\
Band of the second formant of "u" & $900 \sim 1800[\mathrm{~Hz}]$ \\
Band of the first formant of "e" & $300 \sim 700[\mathrm{~Hz}]$ \\
Band of the second formant of "e" & $1500 \sim 2500[\mathrm{~Hz}]$ \\
Band of the first formant of "o" & $350 \sim 650[\mathrm{~Hz}]$ \\
Band of the second formant of "o" & $400 \sim 1300[\mathrm{~Hz}]$ \\
\hline
\end{tabular}

As the suitable order, we adopt the order in the case that the peaks of the spectrum estimate exist without the line splits in the frequency band shown in Table 2 . When analyzing the "A" of Japanese vowels, the upper and lower limits of the appropriate order are shown in Figure 2. From figure 2 , the boundary of the suitable AR order seems to be linear to sampling frequency. In figure 3, approximated boundary lines by least square method are shown.

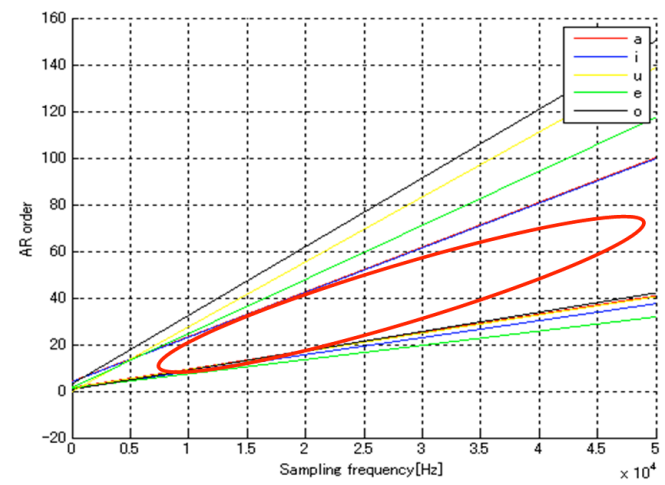

Fig 3 Approximated boundary line between the AR model order and sampling frequency for Japanese vowels

From Figure 3, it can be seen that the proper order may be selected from the region enclosed by the red line. This is also supported from the consideration of Kelly's speech production model. Equations (8) and (9) show the relationship between vocal tract length and length of one section of acoustic tube in Kelly's speech production model.

$$
\begin{aligned}
& L=d \cdot p \\
& d=\frac{c}{2 F s}
\end{aligned}
$$

where $L$ is length of vocal tract, $d$ is length of an acoustic tube, $p$ is AR order, $c$ is speed of sound, and

$F S$ is sampling frequency[5]. From these equations, the next equation can be driven as the relationship between sampling frequency and the AR order.

$$
p=\frac{2 L}{c} F s
$$

In figure 4 , above relationship is added on figure 3, Here, $L$ is assumed $165[\mathrm{~mm}]$ which is common vocal length of male. In this figure, in this figure, equation (10) is represented by red line, green and blue lines are respectively approximate upper boundary and low boundary. From figure 4, we can see that the relationship (10) exists between boundary which we proposed above.

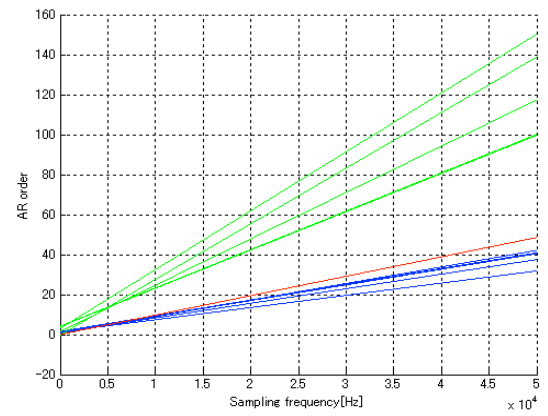


Fig. 4 AR model order and number of the acoustic tube in Kelly's model for sampling frequency

From the above discussion, when AR model order is set to be the number of acoustic tube in Kelly's model, it was found to obtain available estimate of power spectrum for identification of phoneme. Consequently, we select the order shown in Table 3.

Table 3 Selected AR order and sampling frequency

\begin{tabular}{c|c}
\hline \hline Sampling frequency & AR order \\
\hline $25[\mathrm{kHz}]$ & 24 \\
\hline
\end{tabular}

\section{Cluster analysis of the vocal tract area function \\ 3.1 k-means method}

We verify whether it is possible to extract phonological feature from vocal tract area function, through cluster analysis of vocal tract area function coefficients which were estimated from the AR model. Here, k-means method is used. In k-means method, the clusters are generated so as to minimize the objective function shown in (11).

$$
J=\sum_{i=1}^{k} \sum_{x \in G_{i}}\left\|x-v_{i}\right\|^{2}
$$

where $x$ is data of object of cluster analysis, $v_{i}(i=1,2, \ldots k)$ is cluster center, $G_{i}(i=1,2, \ldots k)$ is cluster. Data that you want to cluster analysis is obtained as follows. Vowel speech signal uttered by a subject is divided into frames. Coefficients of the vocal tract area function are estimated from the speech of each frame. Here, 24 coefficients are obtained for each frame signal, where 24 is equal to the order of the AR model. Vocal tract area functions for the five vowels of Japanese are obtained through above process.

\subsection{Experiment and the result of cluster analysis}

Setup condition of experiments is the same one shown in Table 3. For the two subjects, we have conducted an experiment for speech signal acquisition. Cluster analysis was performed for the entire audio signal of five vowels whose sample values are composed of a 24-dimensional vocal tract area function. Upon cluster analysis, the number of clusters is set to 5 , and the initial cluster center is set at random whenever clustering is performed. Setup of cluster analysis is shown in Table 4.

Table 4 Setup of cluster analysis

\begin{tabular}{c|c}
\hline Class & 5 \\
\hline Speech signal & "a", "i", "u", "e", "o" \\
\hline Subject & 2 \\
\hline Number of runs & 100 \\
\hline Dimensions number of cluster & 24 \\
\hline
\end{tabular}

Function coefficients vocal tract area so only shows the ratio of the cross-sectional area of the acoustic tube that make up Kelly's model, it considers two ways to restore the cross-sectional area of the vocal tract. Based on the area of the opening of the mouth of each vowel, we restore the vocal tract area by multiplying its area in order from $A_{0}$. Based on the area of the vocal cord, we restore vocal tract area by multiplying it in reverse order from the $A_{23}$. Table 5 shows the number of original data for the vowel and the number of data in the cluster corresponding to it after cluster analysis.

Table 5 Results of cluster analysis of each subject

\begin{tabular}{|c|c|c|c|c|c|}
\hline Phoneme & "a" & "ij" & "u" & "e" & "o" \\
\hline Original data & 243 & 243 & 243 & 243 & 243 \\
\hline (B) & 243 & 243 & 243 & 243 & 243 \\
\hline Cluster result & 243 & 201 & 227 & 301 & 243 \\
\hline Lips starting point (B) & 252 & 364 & 240 & 125 & 234 \\
\hline Cluster result $\quad$ (A) & $\overline{306}$ & 243 & 180 & 306 & 180 \\
\hline Vocal cord start & 242 & 243 & 254 & 244 & 232 \\
\hline
\end{tabular}

(A) and (B) are attached to distinguish between subjects

The above results can suggest the possibility of identifying the phoneme by the vocal tract area function. But we need to develop more appropriate pretreatment for cluster analysis.

Here, we will consider about which parts of the vocal tract area are available to identify the phoneme. Distribution of the vocal tract area function displays in three dimensions for visually clarity. Examples of vocal tract area function distribution of before and after cluster analysis is shown in figure 5 .
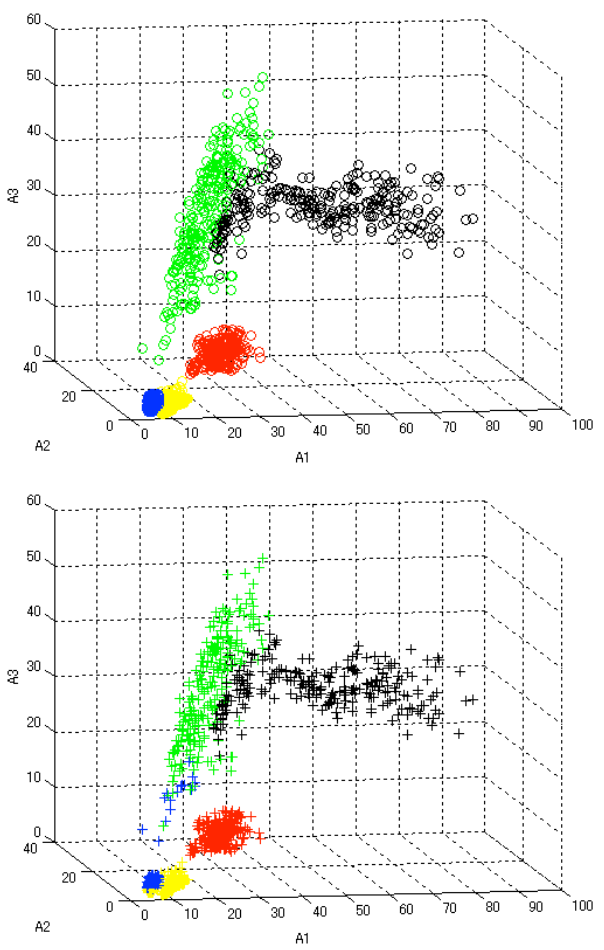

Fig. 5 Distribution of the vocal tract area function 
(above: before analysis, below: after analysis)

In this figure, red, blue, green, yellow, and black denote "a", "i", ,u", "e", and "o" respectively. Superimposed vocal tract area function distribution of results before and after cluster analysis of subject $A$ is shown in figure 6 to figure 8 . Starting point of vocal tract area function distribution of figure 6 to figure 8 is lips.

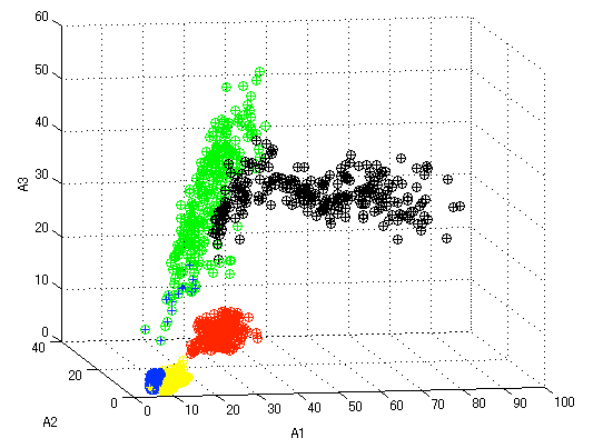

Fig 6 Distribution of the vocal tract area function superimposed (where distance from lips is position of 0.7 to $2.1[\mathrm{~cm}])$

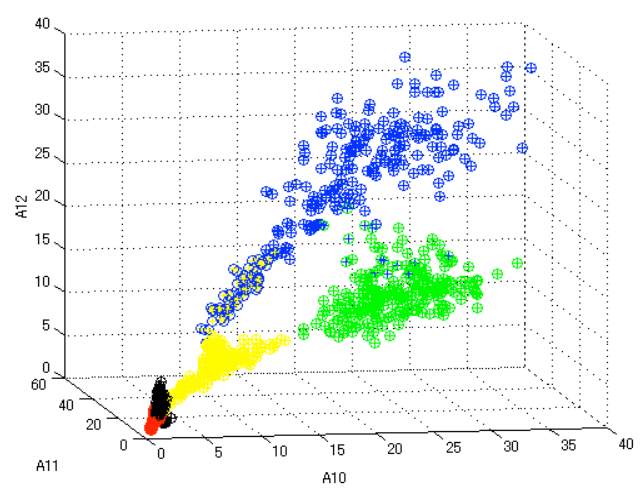

Fig 7 Distribution of the vocal tract area function superimposed (where distance from lips is position of 7.1 to $8.5[\mathrm{~cm}])$

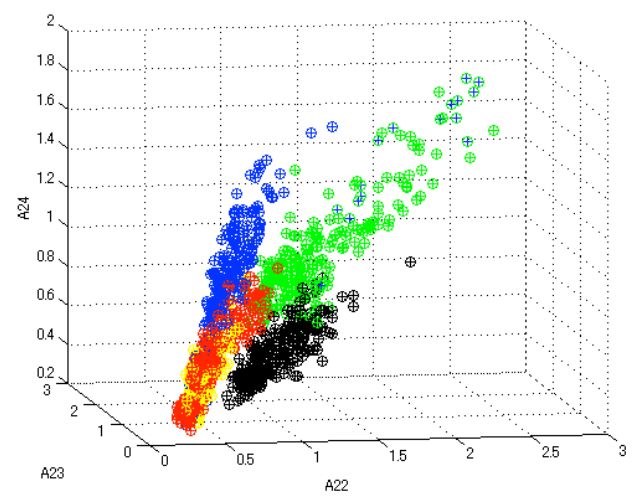

Fig 8 Distribution of the vocal tract area function superimposed (distance from lips is position of 15.6 to $17[\mathrm{~cm}])$

Figure 6 shows the cross-sectional area seems to depend on the phoneme in the oral cavity has been uttered from Fig.6. But figure 8 shows the clusters produced by vocal area function near the vocal cord are close together. Cluster center position of vocal tract area function distribution of figure 6 to figure 8 is shown in Table 7.

Table 7 Cluster center position of the cluster in figure 6,7 , and 8

\begin{tabular}{|c|c|c|c|c|c|}
\hline $\begin{array}{l}\text { Vocal tract } \\
\text { area function }\end{array}$ & "a" & "i" & "u" & "e" & "o" \\
\hline \multirow{3}{*}{$\begin{array}{l}\text { from vocal tract } \\
\text { area coefficients } \\
\text { from } 1 \text { st to } 3 \text { rd }\end{array}$} & 22.4 & 23.2 & 23.2 & 51.2 & 9.66 \\
\hline & 8.78 & 5.25 & 5.25 & 14.5 & 1.62 \\
\hline & 35.6 & 10.2 & 10.2 & 33.7 & 2.14 \\
\hline \multirow{3}{*}{$\begin{array}{l}\text { from vocal tract } \\
\text { area coefficients } \\
\text { from } 10 \text { th to } 12 \text { th }\end{array}$} & 24.4 & 0.90 & 0.90 & 1.60 & 7.24 \\
\hline & 21.7 & 1.40 & 1.40 & 2.26 & 8.62 \\
\hline & 13.4 & 1.82 & 1.82 & 2.95 & 8.63 \\
\hline \multirow{3}{*}{$\begin{array}{l}\text { from vocal tract } \\
\text { area coefficients } \\
\text { form } 22 \text { nd to } 24 \text { th }\end{array}$} & 1.29 & 0.47 & 0.47 & 0.96 & 0.44 \\
\hline & 1.24 & 0.44 & 0.44 & 0.73 & 0.47 \\
\hline & 0.99 & 0.54 & 0.54 & 0.58 & 0.49 \\
\hline
\end{tabular}

From Table 7, cluster center position of each phoneme of vicinity of vocal cords could be confirmed to be in close distance as compared to other. Therefore, it is found that can be classified in each phoneme even if coefficients of vocal tract area function of vicinity of vocal cord excluded.

\section{Conclusions}

In this paper, we have confirmed that the proper AR model order can be selected based on both of power spectrum and Kelly's speech production model. Vocal tract area functions estimated from speech signal are classified by k-means method. As a result, vocal tract area function of 24 dimensions could be classified into each phoneme, if subject of one person. However, it is necessary to examine starting position of vocal tract area function for cluster analysis. When focus on the phonological, it seemed that there is no need to use the number of vocal tract area function dimensions of all for the analysis. It will be needed extraction of vocal tract area function suitable for phoneme recognition.

\section{References}

[1]Yoshinori UCHIMURA, Hideki BANNO and Fumitada ITAKURA, "Analysis of the vocal tract area function aimed at manipulation of voice quality", SLP IPSJ Technical Report, SLP, Spoken Language Processing, 2008(12), 127-132, 2008-02-08

[2]Hayato KINOSHITA and Jun TOYAMA, "Speech Synthesis Using a Contrained Acoustic Tube Model", Institute of Electronics, Information and Communication Engineers, IEICE Technical Report, SP2008-57 (2008-10)

[3]Kohichi OGATA and Yorinobu SONDA, "Articulatory Speech Synthesis System : Development of a GUI-based Interactive System", Information and Communication Engineers, IEICE Technical Report, SP2002-76 (2002-08)

[4]S. Furui"New Acoustic Audio Engineering"(2006) in Japanese [5]IEICE "Hearing and speech" (2006), in Japanese 